\title{
Prognosis of advanced hepatocellular carcinoma patients enrolled in clinical trials can be classified by current staging systems
}

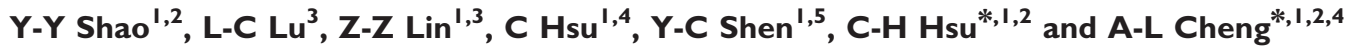 \\ 'Department of Oncology, National Taiwan University Hospital, 7 Chung-Shan S. Road, Taipei 10002, Taiwan; 'ªraduate Institute of Oncology, College of \\ Medicine, National Taiwan University, I Sec. I, Ren'ai Road, Taipei 1005I, Taiwan; ${ }^{3}$ Department of Oncology, National Taiwan University Hospital, \\ Yun-Lin Branch, 95 Xuefu Road, Huwei Township, Yunlin County 63252, Taiwan; ${ }^{4}$ Department of Internal Medicine, National Taiwan University Hospital, \\ 7 Chung-Shan S. Road, Taipei 10002, Taiwan; ${ }^{5}$ Department of Medical Research, National Taiwan University Hospital, 7 Chung-Shan S. Road, Taipei \\ 10002, Taiwan
}

BACKGROUND: Patients enrolled in clinical trials of advanced hepatocellular carcinoma (HCC) are usually required to have good liver reserve and organ function. However, their outcomes are still highly variable. We aimed to examine whether current staging systems can predict the survival of these highly selected patients.

METHODS: Patients from clinical trials involving first-line anti-angiogenic therapy were assigned to different stage groups using the American Joint Committee on Cancer (AJCC), Barcelona Clinic Liver Cancer (BCLC), China integrated score, Cancer of the Liver Italian Program (CLIP) score, Chinese University Prognostic Index (CUPI), Groupe d'Etude et de Traitement du Carcinome Hepatocellulaire (GETCH), Japan Integrated Staging (IIS) score, Okuda, Tokyo score, and a new staging system recently proposed. Survival prediction by the 10 systems was then compared by both univariate and multivariate analyses.

RESULTS: A total of 157 patients were selected for this study. In univariate analysis, all staging systems can predict patient survival except AJCC, BCLC, and JIS score. Concordance indexes for CLIP score, CUPI, and GETCH (0.752, 0.775, and 0.79I, respectively) were significantly higher than those obtained for other staging systems. In multivariate analysis, the CLIP score and CUPI $(P<0.00$ I and 0.009 , respectively) predicted survival more accurately than did the other tested staging systems. Hepatitis B infection and poor performance status were also associated with poor survival.

CONCLUSION: Several HCC staging systems, especially the CLIP score and CUPI, can predict prognosis of patients who are enrolled in clinical trials of advanced HCC.

British Journal of Cancer (2012) I 07, 1672-1677. doi:I0.1038/bjc.2012.466 www.bjcancer.com

Published online II October 2012

(C) 2012 Cancer Research UK

Keywords: clinical trials; hepatocellular carcinoma; prognosis; staging; survival

\begin{abstract}
Advanced hepatocellular carcinoma (HCC) had been a disease with no proven treatment until sorafenib was demonstrated to provide survival benefits in two randomised studies in 2007 (Llovet et al, 2008; Cheng et al, 2009). However, the efficacy of sorafenib is modest. Novel compounds either alone or in combination with sorafenib have been actively explored in phase II or even phase III studies (Hsu et al, 2010a, b; Shao et al, 2010; Cheng et al, 2011).

A staging system that can accurately predict prognosis is crucial for clinical trial designs. For phase II studies, such a system can aid in the selection of a relatively homogeneous yet representative patient population and in the comparison of efficacies across different studies. For phase III studies, such a system can facilitate proper patient stratification and ensure that patient characteristics between treatment arms are balanced. All the commonly used staging systems for HCC were developed in the pre-sorafenib era, and none of them were designed specifically for advanced disease (Okuda et al, 1985; The Cancer of the Liver Italian Program
\end{abstract}

*Correspondence: Dr C-H Hsu; E-mail: chihhunghsu@ntu.edu.tw or Dr A-L Cheng; E-mail: alcheng@ntu.edu.tw

Revised 10 September 2012; accepted 18 September 2012; published online I| October 2012 investigators, 1998; Chevret et al, 1999; Llovet et al, 1999; Green et al, 2002; Leung et al, 2002b; Kudo et al, 2003; Tateishi, 2005; Zhang et al, 2010). Although a new staging system was proposed by Tournoux-Facon et al specifically for patients with HCC in palliative setting (Tournoux-Facon et al, 2011), this new system has not been thoroughly compared with staging systems commonly used in East Asia, such as Chinese University Prognostic Index (CUPI), Japan Integrated Staging (JIS) scores, and China integrated score (CIS).

Two prior studies evaluated the ability of commonly used staging systems to predict the prognosis of patients with advanced HCC (Collette et al, 2008; Huitzil-Melendez et al, 2010). One study found that the Groupe d'Etude et de Traitement du Carcinome Hepatocellulaire (GETCH) predicted patients' prognosis more accurately than did other staging systems at a referral centre in the Unites States. The study did not focus on the clinical trial population (Huitzil-Melendez et al, 2010). The other study demonstrated that the Cancer of the Liver Italian Program (CLIP) score more accurately predicted prognosis than Okuda and Barcelona Clinic Liver Cancer (BCLC) for patients who received either supportive care, tamoxifen alone, or tamoxifen in combination with transarterial chemoembolisation (TACE) (Collette et al, 2008). This study did not include the GETCH and CUPI staging 
systems for analysis, and the treatment is very different from the current standards. Therefore, it remains unclear which of these commonly used staging systems can most accurately predict the prognosis for patients with advanced HCC who are enrolled in clinical trials.

Currently, most clinical trials of advanced HCC use BCLC and Child-Pugh scores to select patients. However, the survival outcomes of these 'well-selected' patients are highly variable. We hypothesised that certain current HCC staging systems can still predict prognosis of these patients, who were enrolled in clinical trials for advanced HCC. The current study was thus conducted to examine the prognosis-predicting performance of 10 staging systems, including the American Joint Committee on Cancer (AJCC; 6th edition) (Green et al, 2002), BCLC (Llovet et al, 1999), CIS (Zhang et al, 2010), CLIP score (The Cancer of the Liver Italian Program investigators, 1998), CUPI (Leung et al, 2002b), GETCH (Chevret et al, 1999), JIS score (Kudo et al, 2003), Okuda (Okuda et al, 1985), Tokyo score (Tateishi, 2005), and the staging system proposed by Tournoux-Facon et al (2011).

\section{MATERIALS AND METHODS}

\section{Study population and variables}

All patients who were enrolled in clinical trials that involved firstline systemic therapy for advanced HCC from May 2005 to June 2010 at National Taiwan University Hospital (NTUH), Taipei, Taiwan, were included in this study. All these studies targeted HCC patients with metastatic or locally advanced disease not amenable to loco-regional therapies, including surgery, TACE, and local ablation. All patients were required to have adequate liver reserve and organ function, good performance status, and measurable lesions according to RECIST criteria (version 1.0) (Therasse et al, 2000). Treatment regimens included either bevacizumab plus capecitabine, sorafenib plus tegafur/uracil, thalidomide plus tegafur/uracil, sorafenib, or sunitinib (Hsu et al, 2010a, b; Cheng et al, 2011; Shao et al, 2012).

Data regarding patient characteristics, laboratory examination results, and overall survival (OS) were retrieved from the original study records. All patients were assessed following the rules (summarised in Supplementary Table 1) of the AJCC (6th edition) (Green et al, 2002), BCLC (Llovet et al, 1999), CIS (Zhang et al, 2010), CLIP score (The Cancer of the Liver Italian Program investigators, 1998), CUPI (Leung et al, 2002b), GETCH (Chevret et al, 1999), JIS score (Kudo et al, 2003), Okuda (Okuda et al, 1985), Tokyo score (Tateishi, 2005), and the staging system proposed by Tournoux-Facon et al (2011). This study was approved by the Institute Research Ethical Committee of NTUH.

\section{Statistical methods}

Statistical analyses were performed with the SAS statistical software (version 9.1.3, SAS Institute Inc., Cary, NC, USA). In statistical testing, a two-sided $P$-value $\leqslant 0.05$ was considered statistically significant. The prognostic predictions of different staging systems were compared univariately by two methods. First, the Kaplan-Meier method was used to estimate OS. For every staging system, OS was compared between every stage group using the log-rank test. Second, concordance (c) indexes were calculated for all staging systems according to the accuracy of their prediction of OS rankings and then compared with each other.

The Cox's proportional hazard model was utilised to compare the 10 staging systems while adjusting other variables with a potential impact on OS. These variables included treatment regimens, age, gender, hepatitis aetiology (hepatitis B virus (HBV) or hepatitis C virus (HCV)), Karnofsky performance scale, and the presence of prior treatment. Staging systems were compared with one another using a model that involved a stepwise variable selection procedure in which the significance levels for entry and significance levels for stay were set to $\geqslant 0.15$. Values of $R^{2}$ and Akaike information criterion (AIC) representing the accuracy of the OS prediction were then calculated for each staging system while adjusting for the confounding variables found by the Cox's model. Higher $R^{2}$ or lower AIC mean better prediction of OS.

\section{RESULTS}

\section{Patient characteristics}

A total of 157 patients, with a median age of 56 years, were included in the current study. Patients received one of the following regimens as first-line therapy for advanced HCC: bevacizumab plus capecitabine $(n=20)$, sorafenib plus tegafur/ uracil $(n=68)$, thalidomide plus tegafur/uracil $(n=34)$, sorafenib $(n=15)$, or sunitinib $(n=20)$. The patient characteristics are summarised in Table 1. Eighty-six percentage of patients were male; 75\% were seropositive for HBV surface antigen (HBsAg); $16 \%$ were seropositive for antibody against HCV (anti-HCV); and $92 \%$ had either extrahepatic metastasis or macroscopic vascular invasion. Except for one patient with Child-Pugh B (score=7) liver reserve, all others were classified to have Child-Pugh A liver reserve. All patients had Karnofsky performance scale indexes $\geqslant 70 ; 120(76 \%)$ patients had a Karnofsky performance scale index $\geqslant 90$.

Table I Patient characteristics

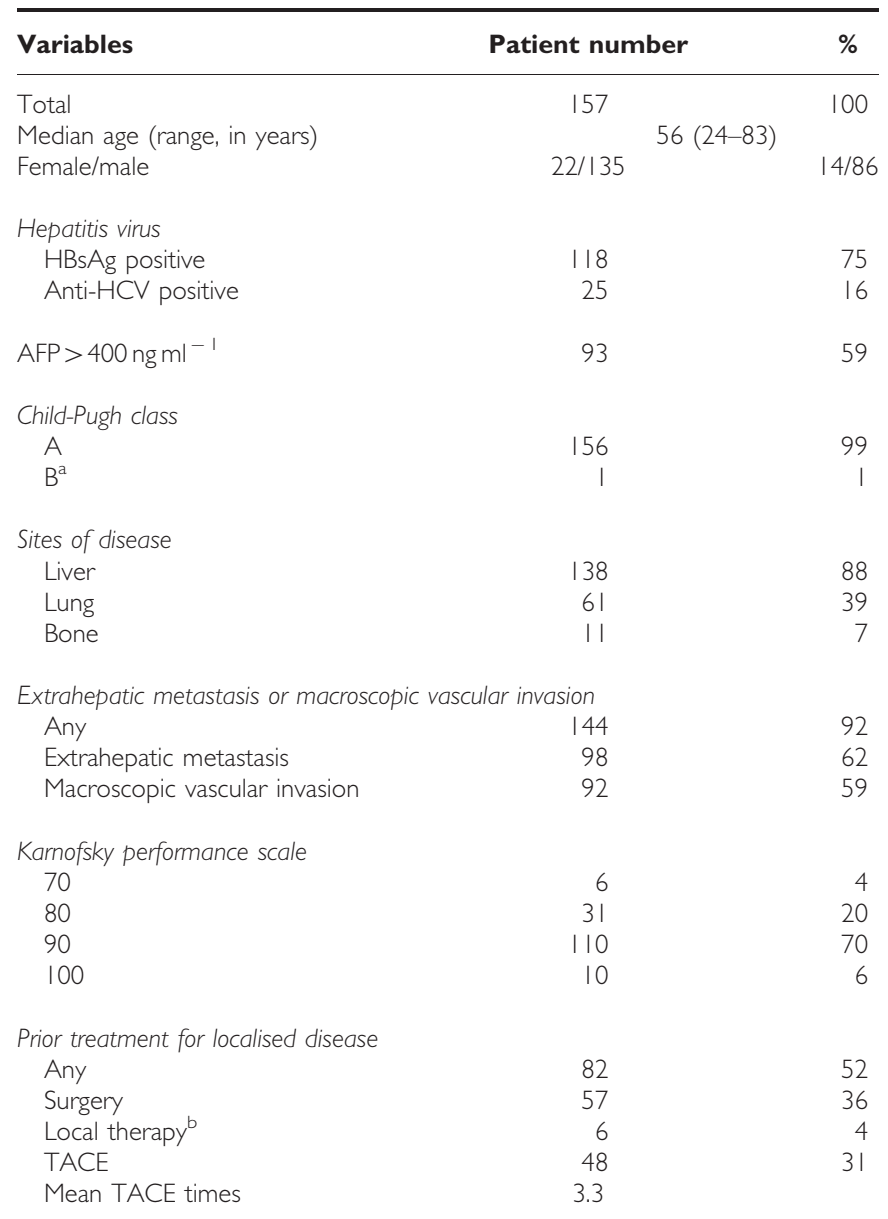

Abbreviations: $\mathrm{AFP}=\alpha$-fetoprotein; $\mathrm{HBsAg}=$ hepatitis $\mathrm{B}$ surface antigen; $\mathrm{HCV}=$ hepatitis $C$ virus; $T A C E=$ transcatheter arterial chemoembolisation. ${ }^{2}$ The Child-Pugh score was 7. Included percutaneous ethanol injection and radiofrequency ablation. 
Patients were classified into stage groups according to 10 staging systems. The distribution of patients among the stage groups is presented in Table 2. As the study focused on patients with advanced HCC enrolled in clinical trials, no patients with early and surgically resectable cases such as AJCC stage I or BCLC stage A were included. Nine (6\%) patients had AJCC stage II disease and 11 (7\%) patients had BCLC stage B disease. These patients had disease either refractory to TACE or not amenable for TACE owing to hypovascularity. The clinical trials also excluded patients with endstage disease or severe liver dysfunction. Therefore, none of the patients were classified as CLIP score $\geqslant 5$, BCLC stage D, Okuda stage III, or high risk according to the staging system proposed by

Table 2 Patient distribution of stage groups

\begin{tabular}{|c|c|c|}
\hline Variables & Patient number & $\%$ \\
\hline \multicolumn{3}{|l|}{ AJCC } \\
\hline$\|$ & 9 & 6 \\
\hline III & 50 & 32 \\
\hline IV & 98 & 62 \\
\hline \multicolumn{3}{|l|}{$B C L C$} \\
\hline B & 11 & 7 \\
\hline C & 146 & 93 \\
\hline \multicolumn{3}{|l|}{ CIS } \\
\hline 0 & 24 & 15 \\
\hline I & 33 & 21 \\
\hline 2 & 41 & 26 \\
\hline 3 & 58 & 37 \\
\hline 4 & 1 & \\
\hline \multicolumn{3}{|l|}{ CLIP score } \\
\hline 0 & 15 & 10 \\
\hline 1 & 32 & 20 \\
\hline 2 & 33 & 21 \\
\hline 3 & 39 & 25 \\
\hline 4 & 38 & 24 \\
\hline \multicolumn{3}{|l|}{ CUPI } \\
\hline Low risk & 48 & 31 \\
\hline Intermediate risk & 80 & 51 \\
\hline High risk & 29 & \\
\hline \multicolumn{3}{|l|}{ GETCH } \\
\hline Low risk & 26 & 17 \\
\hline Intermediate risk & 130 & 83 \\
\hline High risk & 1 & \\
\hline \multicolumn{3}{|l|}{ JIS score } \\
\hline । & 2 & I \\
\hline 2 & 21 & 13 \\
\hline 3 & 134 & 85 \\
\hline \multicolumn{3}{|l|}{ Okuda } \\
\hline 1 & 87 & 55 \\
\hline ॥ & 70 & 45 \\
\hline \multicolumn{3}{|l|}{ Tokyo score } \\
\hline 2 & 23 & 15 \\
\hline 3 & 6 & 4 \\
\hline 4 & 37 & 24 \\
\hline 5 & 87 & 55 \\
\hline 6 & 4 & \\
\hline \multicolumn{3}{|c|}{ The system of Tournoux-Facon et al } \\
\hline Low risk & 123 & 78 \\
\hline Intermediate risk & 34 & 22 \\
\hline
\end{tabular}

Abbreviations: $\mathrm{AJCC}=$ American Joint Committee on Cancer, $\mathrm{BCLC}=$ Barcelona Clinic Liver Cancer; $\mathrm{CIS}=$ China integrated score; CLIP = Cancer of the Liver Italian Program; CUPI = Chinese University Prognostic Index; GETCH = Groupe d'Etude et de Traitement du Carcinome Hepatocellulaire; JIS = Japan Integrated Staging Score.
Tournoux-Facon et al. Interestingly, patients with different CLIP scores were more evenly distributed, with $10 \%, 20 \%, 21 \%, 25 \%$, and $24 \%$ of patients having CLIP scores of $0,1,2,3$, and 4 , respectively.

\section{Survival comparisons among stage groups}

As of 31 December 2010, 138 (88\%) patients had died with a median follow-up time of 35.1 months. Only two patients lost follow-up. The median OS of all patients was 6.6 months $(95 \%$ confidence interval, 5.3-7.9 months). Kaplan-Meier analysis was utilised to estimate the OS, and the log-rank test was used to univariately compare the survival of every stage group (Figure 1). The CIS $(P<0.001)$, CLIP score $(P<0.001)$, CUPI $(P<0.001)$, GETCH $(P<0.001)$, Okuda $(P<0.001)$, Tokyo score $(P<0.001)$, and the staging system of Tournoux-Facon et al $(P<0.001)$ differentiated OS by their stage grouping, whereas the AJCC $(P=0.133)$, BCLC $(P=0.269)$, and JIS score $(P=0.327)$ failed to do so. Notably, patients with CIS scores $=2$ had better survival than patients with CIS scores $=1$.

$\mathrm{C}$ indexes were calculated for all the staging systems. The GETCH, CUPI, CLIP score, Okuda, and the staging system proposed by Tournoux-Facon et al had the highest $\mathrm{c}$ indexes $(0.792,0.775,0.752,0.723$, and 0.710 , respectively), which were not significantly different from one another (Table 3 ). The AJCC, CIS, and BCLC had the lowest $c$ indexes $(0.576,0.546$, and 0.535, respectively, Table 3 ).

To adjust for variables that were less frequently incorporated into staging systems but may also have a prognostic impact on survival, we analysed all staging systems along with these variables in the multivariate analysis, including treatment regimens, age, gender, serum HBsAg, serum antibody against HCV, Karnofsky performance scale, and the presence of prior treatment for localised disease. In the final model, the CLIP score and CUPI emerged as the most accurate predictors of OS $(P<0.001$ and 0.009 , respectively, Table 4$)$. Hepatitis $B$ virus infection and poor performance status were also found to predict poor OS. Adjusting for these two confounding factors, we found that the CLIP score and CUPI yielded the highest $R^{2}$ values $(0.2938$ and 0.1950 , respectively) and the lowest AIC (1134.9 and 1155.5, respectively) for predicting OS (Table 3 ).

\section{DISCUSSION}

This study demonstrated that the CLIP score and CUPI can better predict survival of patients with advanced HCC who had been enrolled in clinical trials using anti-angiogenic agents as first-line therapy. This is the first study specifically focusing on such a patient population. The results can be used in the design of future clinical trials for the treatment of advanced HCC. Although all patients were selected by the eligibility criteria of clinical trials to ensure good liver reserve (99\% Child-Pugh A) and performance status, these two staging systems could successfully differentiate the survival outcome within their stage groups. Their prognostic prediction was better than other systems as determined by different statistical analyses.

Although the study described here was a retrospective analysis, most items in the staging systems examined were prospectively collected upon patient enrolment in the clinical trials. Survival results were mature and very few patients lost follow-up. However, the results may be biased because the study only included patients from one institute. Nevertheless, such a bias should be limited because the selection criteria used in this study were generally consistent with those commonly used in other clinical trials of systemic therapy for advanced HCC.

Previous studies examined survival of patients with advanced HCC in a heterogeneous patient population. Treatment ranged 

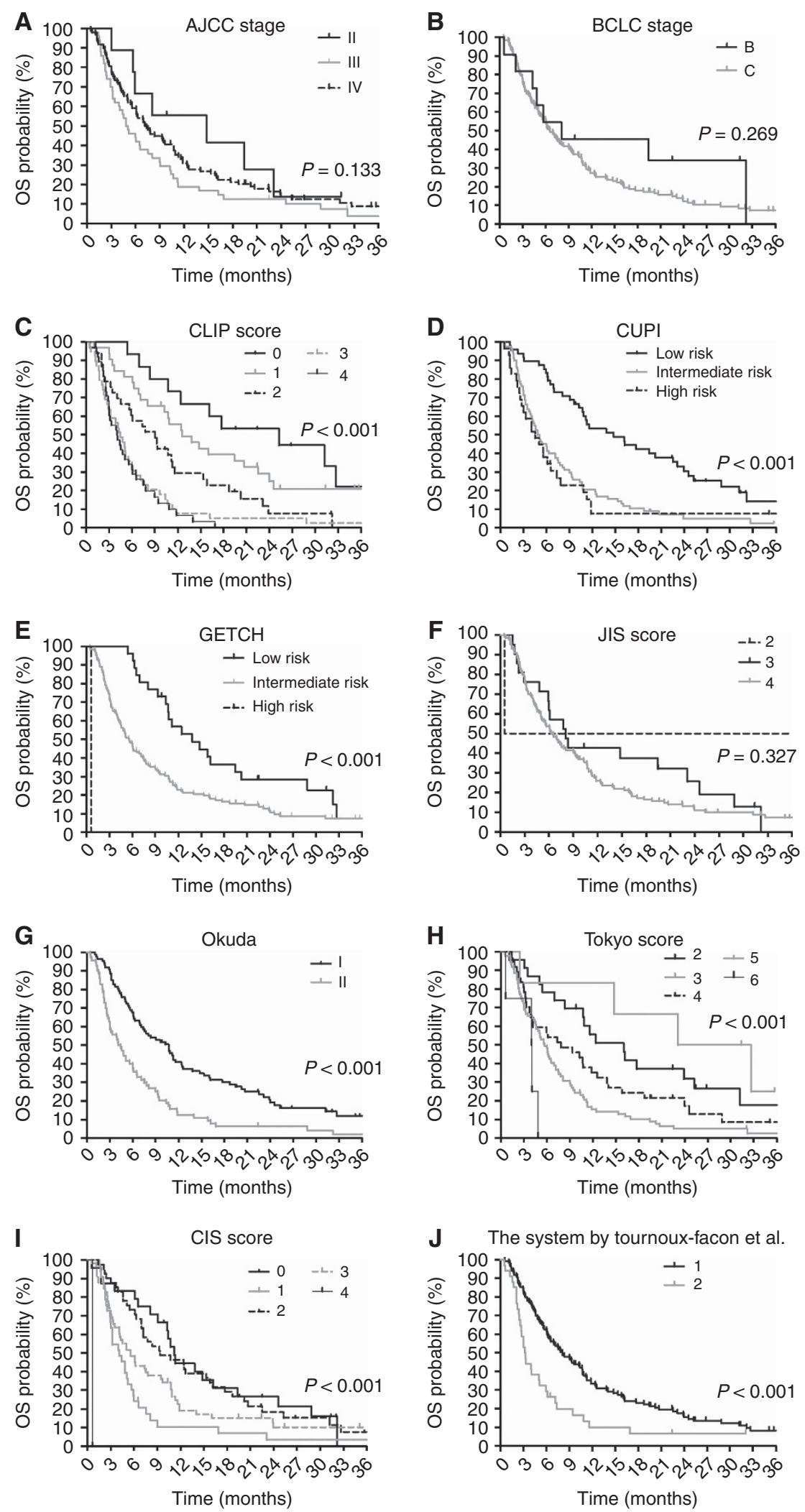

Figure I Kaplan-Meier analysis of overall survival (OS) by every stage group. (A) American Joint Committee on Cancer (AJCC), (B) Barcelona Clinic Liver Cancer (BCLC), (C) Okuda, (D) Cancer of the Liver Italian Program (CLIP) score, (E) Groupe d'Etude et de Traitement du Carcinome Hepatocellulaire (GETCH), (F) Chinese University Prognostic Index (CUPI), (G) Japan Integrated Staging (IIS) Score, (H) Tokyo score, (I) China integrated score (CIS), and (J) the system proposed by Tournoux-Facon et al P-values by log-rank test. 
Table 3 Concordance indexes, $R^{2}$ and AIC of staging systems for their prediction of overall survival

\begin{tabular}{lccc}
\hline & Concordance index & $\mathbf{R}^{\mathbf{2}}$ & AIC \\
\hline GETCH & 0.792 & 0.1134 & $\mid 170.6$ \\
CUPI & 0.775 & 0.1950 & $\mid 155.5$ \\
CLIP score & 0.752 & 0.2938 & 1134.9 \\
Okuda & 0.723 & 0.1577 & $\mid 162.6$ \\
The system of Tournoux-Facon et al & 0.710 & 0.0922 & 1183.1 \\
Tokyo score & 0.678 & 0.1710 & $\mid 160.1$ \\
JIS score & 0.584 & 0.0502 & 1181.4 \\
BCLC & 0.576 & 0.0507 & $\mid 190.1$ \\
CIS & 0.546 & 0.0467 & $\mid 183.5$ \\
AJCC & 0.535 & 0.0429 & $\mid 182.6$ \\
\hline
\end{tabular}

Abbreviations: $\mathrm{AIC}=$ Akaike information criterion; $\mathrm{AJCC}=$ American Joint Committee on Cancer; $\mathrm{BCLC}=$ Barcelona Clinic Liver Cancer; $\mathrm{CIS}=$ China integrated score: CLIP = Cancer of the Liver Italian Program; CUPI = Chinese University Prognostic Index; GETCH = Groupe d'Etude et de Traitement du Carcinome Hepatocellulaire: JIS = Japan Integrated Staging Score.

Table 4 Final Cox's proportional hazards model ${ }^{a}$ for best staging systems to predict overall survival

\begin{tabular}{lrcc}
\hline Variables & P-value & Hazard ratio & $\mathbf{9 5 \%} \mathbf{~ C l}$ \\
\hline HBsAg $(+)$ & 0.016 & 1.652 & $1.099-2.484$ \\
Karnofsky performance scale & 0.024 & 0.969 & $0.944-0.996$ \\
CUPI & 0.004 & 1.853 & $1.215-2.828$ \\
CLIP score & $<0.001$ & 1.671 & $1.371-2.037$ \\
\hline
\end{tabular}

Abbreviations: $\mathrm{Cl}=$ confidence interval; CLIP = Cancer of the Liver Italian Program; CUPI = Chinese University Prognostic Index; $\mathrm{HBsAg}=$ hepatitis B virus surface antigen. ${ }^{\mathrm{a}} \mathrm{Co}$-variables included for variable selection were treatment regimens, age, gender, $\mathrm{HBsAg}$, antibody against hepatitis $\mathrm{C}$ virus, Karnofsky performance scale, the presence of prior treatment for localised disease and all the 10 staging systems studied.

from supportive care, TACE, cytotoxic chemotherapy, to targeted therapy (Collette et al, 2008; Huitzil-Melendez et al, 2010; Lin et al, 2012), and patients may not all have been enrolled in clinical trials. These studies found that CLIP score, CUPI, or GETCH can better differentiate prognosis of these patients. Among them, our prior study found CLIP score as a better staging system for patients who received various systemic treatment for advanced HCC (Lin et al, 2012). In the current study, we focused on a patient population that is more relevant to current practice. The results demonstrated that the CLIP score and CUPI emerged as the best systems for predicting OS after adjusting for other potential prognostic factors that are not included in most staging systems. Above all, CLIP could be considered a pivotal stratification factor in clinical trial designs because it was repeatedly demonstrated to predict prognosis of patients enrolled in clinical trials, regardless the treatment regimens.

In addition to the CLIP and CUPI, we found that viral aetiology was a prognostic factor in the multivariate analyses, which is consistent with other reports (Leung et al, 2002a; Cantarini et al, 2006; Chen et al, 2006; Shao et al, 2011). Positive HBsAg was associated with poorer survival (Cantarini et al, 2006; Chen et al, 2006; Shao et al, 2011). As HCC resulting from different aetiological factors can have different carcinogenesis and molecular signatures (Okabe et al, 2000; Laurent-Puig et al, 2001; Iizuka et al, 2002; Moinzadeh et al, 2005), it is not surprising that aetiology should have an impact on prognosis of patients with HCC. On the contrary, several potential prognostic predictors were not identified by the current analysis because some of them (e.g., $\alpha$-fetoprotein) were incorporated into the staging systems, while others were homogenous (e.g., all but one of our patients had Child-Pugh A status) in the entire study population.

In conclusion, our study indicates that several current HCC staging systems, especially CLIP score and CUPI, can predict survival of a highly selected patient cohort consisting of patients who were enrolled in clinical trials of advanced HCC. These two staging systems should be considered when selecting eligibility criteria and/or setting the stratification for randomisation to ensure an optimal clinical trial design.

\section{ACKNOWLEDGEMENTS}

This work was supported by grant NSC98-3112-B-002-038, NSC101-2314-B-002-141, 100CAP1020-2, and NTUH.101-N1965.

Supplementary Information accompanies the paper on British Journal of Cancer website (http://www.nature.com/bjc)

\section{REFERENCES}

Cantarini MC, Trevisani F, Morselli-Labate AM, Rapaccini G, Farinati F, Del Poggio P, Di Nolfo MA, Benvegnu L, Zoli M, Borzio F, Bernardi M (2006) Effect of the etiology of viral cirrhosis on the survival of patients with hepatocellular carcinoma. Am J Gastroenterol 101: 91-98

Chen CH, Huang GT, Yang PM, Chen PJ, Lai MY, Chen DS, Wang JD, Sheu JC (2006) Hepatitis B- and C-related hepatocellular carcinomas yield different clinical features and prognosis. Eur J Cancer 42: 2524-2529

Cheng A, Kang Y, Lin D, Park J, Kudo M, Qin S, Omata M, Pitman Lowenthal SW, Lanzalone S, Yang L, Lechuga M, Raymond E (2011) Phase III trial of sunitinib (Su) versus sorafenib (So) in advanced hepatocellular carcinoma (HCC). J Clin Oncol 29(suppl): abstr 4000

Cheng AL, Kang YK, Chen Z, Tsao CJ, Qin S, Kim JS, Luo R, Feng J, Ye S, Yang TS, Xu J, Sun Y, Liang H, Liu J, Wang J, Tak WY, Pan H, Burock K, Zou J, Voliotis D, Guan Z (2009) Efficacy and safety of sorafenib in patients in the Asia-Pacific region with advanced hepatocellular carcinoma: a phase III randomised, double-blind, placebo-controlled trial. Lancet Oncol 10: 25-34

Chevret S, Trinchet JC, Mathieu D, Rached AA, Beaugrand M, Chastang C (1999) A new prognostic classification for predicting survival in patients with hepatocellular carcinoma. Groupe d'Etude et de Traitement du Carcinome Hepatocellulaire. J Hepatol 31: 133-141

Collette S, Bonnetain F, Paoletti X, Doffoel M, Bouche O, Raoul JL, Rougier P, Masskouri F, Bedenne L, Barbare JC (2008) Prognosis of advanced hepatocellular carcinoma: comparison of three staging systems in two French clinical trials. Ann Oncol 19: 1117-1126

Green FL, Page DL, Fleming ID (eds) (2002) Liver including intrahepatic bile ducts. In AJCC Cancer Staging Handbook. 6th edn pp 131-144. Springer: New York

Hsu CH, Shen YC, Lin ZZ, Chen PJ, Shao YY, Ding YH, Hsu C, Cheng AL (2010a) Phase II study of combining sorafenib with metronomic tegafur/ uracil for advanced hepatocellular carcinoma. J Hepatol 53: 126-131

Hsu CH, Yang TS, Hsu C, Toh HC, Epstein RJ, Hsiao LT, Chen PJ, Lin ZZ, Chao TY, Cheng AL (2010b) Efficacy and tolerability of bevacizumab plus capecitabine as first-line therapy in patients with advanced hepatocellular carcinoma. Brit J Cancer 102: 981-986

Huitzil-Melendez FD, Capanu M, O’Reilly EM, Duffy A, Gansukh B, Saltz LL, Abou-Alfa GK (2010) Advanced hepatocellular carcinoma: which staging systems best predict prognosis? J Clin Oncol 28: 2889-2895

Iizuka N, Oka M, Yamada-Okabe H, Mori N, Tamesa T, Okada T, Takemoto N, Tangoku A, Hamada K, Nakayama H, Miyamoto T, Uchimura S, Hamamoto Y (2002) Comparison of gene expression profiles between hepatitis B virus- and hepatitis $\mathrm{C}$ virus-infected hepatocellular carcinoma by oligonucleotide microarray data on the basis of a supervised learning method. Cancer Res 62: 3939-3944

Kudo M, Chung H, Osaki Y (2003) Prognostic staging system for hepatocellular carcinoma (CLIP score): its value and limitations, and a 
proposal for a new staging system, the Japan Integrated Staging Score (JIS score). J Gastroenterol 38: 207-215

Laurent-Puig P, Legoix P, Bluteau O, Belghiti J, Franco D, Binot F, Monges G, Thomas G, Bioulac-Sage P, Zucman-Rossi J (2001) Genetic alterations associated with hepatocellular carcinomas define distinct pathways of hepatocarcinogenesis. Gastroenterology 120: 1763-1773

Leung TW, Tang AM, Zee B, Yu SC, Lai PB, Lau WY, Johnson PJ (2002a) Factors predicting response and survival in 149 patients with unresectable hepatocellular carcinoma treated by combination cisplatin, interferonalpha, doxorubicin and 5-fluorouracil chemotherapy. Cancer 94: 421-427

Leung TWT, Tang AMY, Zee B, Lau WY, Lai PBS, Leung KL, Lau JTF, Yu SCH, Johnson PJ (2002b) Construction of the Chinese University Prognostic Index for hepatocellular carcinoma and comparison with the TNM staging system, the Okuda staging system, and the Cancer of the Liver Italian Program staging system. Cancer 94: 1760-1769

Lin ZZ, Hsu C, Hu FC, Shao YY, Chang DY, Yang CH, Hong RL, Hsu CH, Cheng AL (2012) Factors impacting prognosis prediction in BCLC Stage $\mathrm{C}$ and Child-Pugh Class A hepatocellular carcinoma patients in prospective clinical trials of systemic therapy. Oncologist 17: 970-977

Llovet JM, Bru C, Bruix J (1999) Prognosis of hepatocellular carcinoma: the BCLC staging classification. Semin Liver Dis 19: 329-338

Llovet JM, Ricci S, Mazzaferro V, Hilgard P, Gane E, Blanc JF, de Oliveira AC, Santoro A, Raoul JL, Forner A, Schwartz M, Porta C, Zeuzem S, Bolondi L, Greten TF, Galle PR, Seitz JF, Borbath I, Haussinger D, Giannaris T, Shan M, Moscovici M, Voliotis D, Bruix J, Group SIS (2008) Sorafenib in advanced hepatocellular carcinoma. N Engl J Med 359: 378-390

Moinzadeh P, Breuhahn K, Stutzer H, Schirmacher P (2005) Chromosome alterations in human hepatocellular carcinomas correlate with aetiology and histological grade - results of an explorative CGH meta-analysis. $\mathrm{Br} \mathrm{J}$ Cancer 92: 935-941

Okabe H, Ikai I, Matsuo K, Satoh S, Momoi H, Kamikawa T, Katsura N, Nishitai R, Takeyama O, Fukumoto M, Yamaoka Y (2000) Comprehensive allelotype study of hepatocellular carcinoma: potential differences in pathways to hepatocellular carcinoma between hepatitis B virus-positive and -negative tumors. Hepatology 31: 1073-1079
Okuda K, Ohtsuki T, Obata H, Tomimatsu M, Okazaki N, Hasegawa H, Nakajima Y, Ohnishi K (1985) Natural history of hepatocellular carcinoma and prognosis in relation to treatment. Study of 850 patients. Cancer 56: 918-928

Shao YY, Chen PJ, Lin ZZ, Huang CC, Ding YH, Lee YH, Hsu CH, Cheng AL (2011) Impact of baseline hepatitis B viral DNA levels on survival of patients with advanced hepatocellular carcinoma. Anticancer Res 31: 4007-4011

Shao YY, Lin ZZ, Hsu C, Lee KD, Hsiao CH, Lu YS, Huang CC, Shen YC, Hsu CH, Cheng AL (2012) Efficacy, safety, and potential biomarkers of thalidomide plus metronomic chemotherapy for advanced hepatocellular carcinoma. Oncology 82: 59-66

Shao YY, Lin ZZ, Hsu C, Shen YC, Hsu CH, Cheng AL (2010) Early alphafetoprotein response predicts treatment efficacy of antiangiogenic systemic therapy in patients with advanced hepatocellular carcinoma. Cancer 116: 4590-4596

Tateishi R (2005) Proposal of a new prognostic model for hepatocellular carcinoma: an analysis of 403 patients. Gut 54: 419-425

The Cancer of the Liver Italian Program investigators (1998) A new prognostic system for hepatocellular carcinoma: a retrospective study of 435 patients. Hepatology 28: 751-755

Therasse P, Arbuck SG, Eisenhauer EA, Wanders J, Kaplan RS, Rubinstein L, Verweij J, Van Glabbeke M, van Oosterom AT, Christian MC, Gwyther SG (2000) New guidelines to evaluate the response to treatment in solid tumors. European Organization for Research and Treatment of Cancer, National Cancer Institute of the United States, National Cancer Institute of Canada. J Natl Cancer Inst 92: 205-216

Tournoux-Facon C, Paoletti X, Barbare JC, Bouche O, Rougier P, Dahan L, Lombard-Bohas C, Faroux R, Raoul JL, Bedenne L, Bonnetain F (2011) Development and validation of a new prognostic score of death for patients with hepatocellular carcinoma in palliative setting. J Hepatol 54: $108-114$

Zhang BH, Wang XH, Yue HY, Ling CQ (2010) A new staging system is more discriminant than conventional staging systems for unresectable hepatocellular carcinoma. J Cancer Res Clin Oncol 136: 821-827

This work is published under the standard license to publish agreement. After 12 months the work will become freely available and the license terms will switch to a Creative Commons Attribution-NonCommercial-Share Alike 3.0 Unported License. 